\title{
Der Wildesche Schnitt und dessen Heilpotenz in der Ohrenheilkunde.
}

\author{
Von
}

Dr. P. Papanikoláou, Konstantinopel.

Zur Behandlung von Warzenfortsatzentzündungen wurde früher unter anderem auch der von Wilde ${ }^{1}$ ) empfohlene und nach ihm genannte Schnitt angewendet. Die neuere Schule steht nicht ganz freundlich zu dem kleinen Eingriff, und ich glaube mit Unrecht.

Jacobson ${ }^{2}$ ) empfiehlt die Wildesche Inzision nur gegen die Periostitis mast. Zeigt sich aber die in größerer Ausdehnung nachher bloßgelegte Kortikalis verfärbt oder fistulös durchlöchert, so schreitet er zur Aufmeißlung vor. Weiter fügt erhinzu :

„Ein eifriger Anhänger desselben (Wildeschen Schnittes), im Gegensatz zu der frühzeitigen Aufmeißlung des Warzenteils, ist Stetter geblieben. Er vollführt bei a 11 e n Periostitiden der Pars mast. infolge von Mittelohreiterung $\mathrm{z} \mathrm{un} \mathrm{ä} \mathrm{chst}$ s t e $t \mathrm{~s}$ die Wildesche Inzision und nur, wenn er den Knochen bereits kariös oder bläulich verfärbt fand, schreitet er zur Fröffnung des Antrunx: Für letztere besteht nach S.'s Ansicht selbst bei dem Vorhandensein einer intrakraniellen Affektion oder den ersten Zeichen einer Hyperämie der Papilla. optica noch keine dringende Indikation. Er hat auch in solchen Fällen durch bloße Eröffnung eines subperiostalen Abszesses. in der Regio mast. noch Heilung erzielt und plädiert daher für folgendes Verfahren: ,Bei allen Periostitiden des Warzenteils infolge von akuter oder chronischer Mittelohreiterung soll die Wildesche Inzision jederzeit der erste operative Eingriff sein. Er genügt oft zur Heilung selbst dann, wenn der Augenhintergrund und die bekannten Allgemeinsymptome eine meningeale Reizung bereits sicher annehmen lassen. Zeigt sich die Kor-

1) Wilde, Ohrenheilkunde, deutsche Übersetzung 1855.

$\left.{ }^{2}\right)$ Jacobson, Lehrbuch der Ohrenheilkunde 1898. 
tikalis makroskopisch verändert, besonders blạu verfärbt und vielleicht schon elastisch federnd, was in ausgesprochener Weise nur bei chronischen Mittelohreiterungen vorkommt, so soll man das Antrum eröffnen, sonst abwarten, ob sich nicht innerhalb der nächsten 8-10 Tage der ganze Zustand wesentlich bessert, und wenn das nicht der Fall ist, ja, wenn vielleicht schon am Tage nach der ersten Operation sich die Symptome verschlimmern, dann sofort zur Aufmeißelung schreiten.' Können wir uns mit diesen Anschauungen auch nicht einverstanden erklären, insbesondere, wenn bereits "Zerebralsymptome' bestehen, so ist es immerhin von nicht geringem Interesse und durfte daher nicht unerwähnt bleiben, daß Stetter durch Wildesche Inzision bereits bei 35 Kranken mit chronischer Mittelohreiterung und Periostitis mast. nicht nur die Periostitis, sondern auch die Mittelohreiterung zu völliger Heilung gebracht hat."

Politzer ${ }^{1}$ ) äußert sich in seinem sehr bekannten Werk sehr zweifelhaft über den Heilwert des Wildeschen Schnittes folgendermaßen: ,Der früher bei der akuten Mastoiditis ausgeführte. Wildesche Schnitt findet jetzt im beschränkten Maße Anwendung. Er besteht in einer bis auf den Knochen reichenden senkrechten, $4-5 \mathrm{~cm}$ langen Inzision durch die in f il t r i er te Bedeckung des Warzenfortsatzes, $1-2 \mathrm{~cm}$ hinter der Insertion der Ohrmuschel. Ich wende den Wildeschen Schnitt jetzt nur bei der primären Periostitis mastoid. an, seltener bei den im Verlaufe genuiner, akuter Mittelohreiterung e $\mathrm{n}$ auftretenden schmerzhaften Schwellungen am Warzenfortsatze in den ersten Tagen der Erkrankung. Hier sieht man nicht selten nach dem. Wildeschen Schnitt die Symptome der Mastoiditis zurückgehen. Hingegen wird man bei ausgesprochenen Symptomen von Abszeßbildungen im Warzenfortsatze, namentlich bei der Influenza-Otitis und bei den Infektionskrankheiten, béi welchen man nach der AufmeiBelung fast im $\mathrm{m}$ er einen Eiterherd im Warzenfortzatze findet, auf den Wildeschen Schnitt verzichten und zur Eröffnung des Warzenfortsatzes schreiten. Daß auch zuweilen bei ausgesprochener Abszeßbildung im Warzenfortsatze nach dem Wildeschen Schnitt der Prozeß sich zurückbildet, beweist, daß Einschmelzungsprozesse im Warzenfortsatze auch ohne operative Eröffnung derselben heilen können. Noch seltener findet der Wildesche Schnitt Anwen-

1) Politzer, Lehrbuch der Ohrenheilkunde 1901. 
dung bei den chronischen Mittelohreiterungen, da hier die anatomischen Veränderungen im Proc. mast. einen Dauererfolg dieses Eingriffes ausschließen."

Noch energischer gegen die Wildesche Inzision tritt Heine ${ }^{1}$ ) auf. ,In der neueren Zeit, schreibt er, sind die Ohrenärzte von dem Wildeschen Schnitt immer mehr abgekommen. Auch in unserer Klinik (Berlin) wird er nicht mehr gemacht. Uns leiten dabei folgende Erwägungen: Hat die eitrige Mittelohrentzündung bereits zu einer erheblichen Erkrankung des Warzenfortsatzes geführt, produziert die erkrankte Schleimhaut der pneumatischen Räume Eiter, oder ist es zu einer eitrigen Entzündung des Knochens selbst gekommen, hat sich gar schon eine größere, mit Eiter und Granulationen gefüllte Höhle gebildet, so werden wir mit einer einfachen Inzision bis auf den Knochen, obschon ein subperiostaler Abszeß besteht oder nicht, nicht ausreichen. Der Prozeß im Warzenfortsatz wird dadurch nicht beeinflußt werden, und über kurz oder lang werden wir zur Eröffnung desselben schreiten müssen . . ."

Körner ${ }^{2}$ ) weist darauf hin, daß bei ganz kleinen Kindern ein wirkliches Empyem des Antrum durch die Fissura mastoideosquamosa nach außen durchbrechen, und daß der Eiter das Periost abheben kann. Inzidiert man diesen Abszeß, so kann ebenso wie bei Spontandurchbruch desselben Heilung eintreten, da es sich ja nicht um eine Knochenerkrankung, sondern nur um eine Eiterverhaltung im Antrum handelt. Bestehen dagegen Herde im Warzenfortsatz, die aus einer Finschmelzung des Knochens hervorgegangen sind, so genügt nach seiner Ansicht ebenfalls der Wildesche Schnitt nicht!

Wie man hieraus ersehen kann, fand jene warme Verteidigung der Wildeschen Inzision seitens Stetters fast keine Nachahmung, so da $B$ der kleine, aber oft segensreiche Eingriff fast in Vergessenheit geraten ist und von neueren Autoren, wie Laurens ${ }^{3}$ ), Lannois ${ }^{4}$ ) usw. nicht mehr erwähnt wird.

Ich bin so glücklich gewesen, den kleinen Eingriff in über 70 Fällen von Mastoiditis acuta mit Periostitis mast. mit gutem Erfolg zu prüfen. Alle diese Fälle bis auf einen, der als Mastoiditis tuberculosa später auch mikroskopisch diagnostiziert wurde, sind damit völlig geheilt worden.

1) Heine, Operationen am Ohr. 1904

2) Körner, Eitrige Erkrankungen des Schläfenbeins.

s) Laurens, Chirurgie Otolaryngologique 1906. 1908.

4) Lannois, Precis des maladies de l'oreille, du nez et du larynx 
Die nach dem Eingriff wahrgenommenen anatomischen Veränderungen waren meistenteils sehr enge Knochenfisteln, oder seltener Zerstörung und Einschmelzung der Kortikalis in einer solchen Ausdehnung, daß eine solche Knochenhöhle sich vorfand, wie wir sie bei der Aufmeißelung zu präparieren gewöhnt sind. Dieselbe wurde mit Eiter und Granulationen voll gefunden; letztere wurden niemals ausgekratzt. Die aufgefundenen Höhlen wurden einfach mit steriler oder Jodoformgaze austamponiert oder durch die vorhandenen Fisteln die Mittelohrräume mit steriler Borsäurelösung täglich solange ausgespült, bis die Ausspülungsflüssigkeit durch diese Räume und den Gehörgang ausfließen konnte. Bei einigen hartnäckigen Fällen war ich genötigt, den Fistelgang mit der Lapisperle mit gutem Erfolg zu ätzen. Jegliche weitere Polypragmasie wurde unterlassen.

In folgendem werde ich nur über einige Fälle, welche Erwachsene betreffen, kurz berichten; dieselben sollen zeigen, daß Mastoiditiden mit Periostitis mast. entgegen den oben erwähnten Ansichten Körners nicht nur bei Kindern, sondern auch bei Erwachsenen durch den Wildeschen Schnitt geheilt werden können.

Bemerkt sei, daß ich diesen Schnitt nicht typisch nach den oben erwähnten Regeln der klassischen Autoren vollführe, sondern in der Mitte und in den Grenzen der vorhandenen Fluktuation und parallel zur Ohrmuschel, so daß die Inzisionslänge $4 \mathrm{~cm}$ nicht überschreitet und bisweilen je nach der Lage des Abszesses mehr nach hinten, unten oder oben liegt. Bei eng begrenzten Fluktuationen führe ich die Spitze des Messers senkrecht durch dieselben bis zum Knochen und fahnde nach vorhandenen Fisteln mit der Sonde.

1. Herr M. K., 45 Jahre alt. Im Februar 1901 litt Pat. an heftigen linksseitigen Ohrenschmerzen, welche ein Kollege mit Tropfen und Blutegeln hinter dem Ohr erfolglos behandelte. Nach spontanem Ohrausfluß ließen die Schmerzen bis Mai nach. Wegen erneuerten Ohrund Kopfschmerzen und einer Druckempfindlichkeit in der Regio mast. sin. wùrde von mir die AufmeiBlung empfohlen, welche vom Pat. abgelehnt wurde. Er wurde nachher von einem dritten Kollegen mit einer Salbe behandelt und dann kehrte er mit sehr heftigen Kopfschmerzen und einer starken fluktuierenden Schwellung hinter dem linken $\mathrm{Ohr}$ wieder zu mir. Inzision nach Wilde brachte eine ansehnliche Menge von Eiter und eine Knochenfistel zu Tage, durch welche eine Sonde $4 \mathrm{~cm}$ tief in die Mittelohrräume eingeführt werden konnte. In 10 Tagen definitive Heilung mit normalem Gehör.

2. Herr B., 60 Jahre alt, 7. II. 1903. Seit 25 Tagen klagt Pat. über heftige Ohrschmerzen rechts infolge von Influenza. Dieselben haben seit. 3 Tagen unter Fieberbewegungen zwischen $37,5^{0}-39,5^{\circ}$ zugenommen; gestern ein Frostanfall, kein OhrausfluB. - Regio mast.

Archiv f. Ohrenheilkunde, Bd. 85. 
stark druckempfindlich. Das vorgewölbte Trommelfell wird breit gespalten. Es folgte eine profuse Ohreiterung, welche mit fleißigen Aspirationen nach meiner') Methode und Ausspïlungen mit steriler Borsäurelösung behandelt wurde, wonach die Schmerzen und das Fieber nachließen. Es blieb aber eine kleine zirkumskripte Stelle an der Warzenfortsatzspitze bis 8. III. druckempfindlich. Hier entwickelte sich jetzt eine eng begrenzte Infiltration der Weichteile, welche durch eine kleine bis zum Knochen gehende Inzision gespalten wurde. Danach sind Mastoidschmerzen und Ohreiterung schnell und für immer beseitigt.

3. Herr A. J., 48 Jahre alt, aus 40 Klisse, exkrankte am Ende August 1903 an einer Halsentzündung mit rechtsseitigen Ohren- und Kopfschmerzen. Nach 15 Tagen entstand eine schmerzhafte Schwellung hinter der rechten Ohrmuschel, welche in 40 Klisse gespalten wurde. Pat. wurde danach zur Operation nach Konstantinopel geschickt. Der stark neurasthenische Mann blieb einige Tage hier und konsultierte jeden Tag 4 Ohrenärzte und 3 Chirurgen. Nur mit großer Mühe konnte ich ihn vor der AufmeiBlung beschützen. Sämtliche Kollegen waren dafür. Pat. fuhr einige Tage später ohne weiteres geheilt nach Hause zurück und blieb, wie ich mich später erkundigte, an seinem Ohr gesund. Ich fand bei seiner ersten Konsultation eine sehr tiefe, wenig eiternde Knochenfistel im Warzenfortsatze und das Trommelfell intakt. Das Ohr hatte sonst niemals geeitert.

4. Herr B. M., 44 Jahre alt, klagt seit 4 Monaten über Gehörschwäche rechts, über Ohrenschmerzen vor 3 Monaten, die nach eingesetzter Ohreiterung nachließen und seit 4 Tagen auf dem rechten Ohre wieder enschienen; vor 3 Tagen einmal Frost. Seit 1 1/2 Monat soll das ohr nicht mehr ausfließen.

30. IV. 1905. Schwellung mit Fluktuation an der rechten Warzenfortsatzgegend, welche inzidiert wird; viel dicker Eiter. Am hinteren Rande der Warzenfortsatzspitze eine ziemlich breite Knochenfistel, durch welche eine Sonde $5 \mathrm{~cm}$ tief in die Warzenfortsatzräume eindringt. In einigen Tagen komplette Heilung.

5. Herr K. J., 16 Jahre alt. 14. III. 1907. Klagt über heftige Ohrschmerzen rechts seit 3-4 Tagen nach Influenza und Schnupfen. Das stark gerötete und vorgewölbte Trommelfell wird gleich inzidiert, wonach profuse Ohreiterung. Warme Umschläge.

18. III. 1907. Schwellung oberhalb der rechten Ohrmuschel und Oedem des rechten unteren Augenlides; Regio mast. leicht gerötet, stark druckempfindlich ohne Fluktuation.

22. III. 1907. Fluktuierende Infiltration am Warzenfortsatze wird nach Wilde inzidiert; reichliche Eiterentleerung durch eine breite und tiefe Kortikalisfistel am Planum mast. Drainage desselben mit Jodoformgaze. Die Nachbehandlung dauerte ungefähr 3 Wochen, wonach das $\mathrm{Ohr}$ definitiv heilte.

6. Herr J. B., 20 Jahre alt, trat am 10. XII. 1907 mit Lungenentzündung ins Österreich-Ungarische Spital ein. Beim Erlöschen der Pneumonie am 17. XII. rechtsseitige Ohrenschmerzen, welche nach spontanem Ohrausflu $B$ nachließen. Die Warzenfortsatzgegend blieb aber weiter druckempfindlich. Diese Druckempfindlichkeit hat hier allmählich zugenommen und es bildete sich ein Abszeh, der am 5. I. 1908 nach Wilde eröffnet wurde. Am Planum mast. wurde eine Knochenfistel aufgefunden, durch welche die Mittelohrräume mit steriler Borsäurelösung ausgespült wurden. Nach 2 Tagen hörte die

$\left.{ }^{1}\right)$ Papanikoláou. Eine neue Methode zur Behandlung von akuten Mittelohrentzündungen. - Biol. Sektion der Griech. phil. Syllogus 20. XII. 1900. 
profuse Ohreiterung auf und einige Tage später heilte auch die retroaurikuläre Wunde definitiv aus.

7. Herr M., 50 Jahre alt, klagt über linksseitige Ohren- und Koptschmerzen, welche seit ungefähr 1 Monat nach Influenza und Schnupfen bestehen; seit 15 Tagen linksseitiger Ohrenfluß.

l. III. 1908. Uhr a. 1. hörbar, Planum mast. druckempfindlich; zitzenförmige Perforation links hinten oben wird erweitert und das Sekret 20 Tage lang fleiBig, aber enfolglos aspiriert; Temperatur $36,5^{\circ}$ bis $37,5^{\circ}$.

28. III. 1908. Mit zunehmender Druckempfindlichkeit auf der Regio mast. entsteht eine rote, glänzende Schwellung, welche die linke Temporal-Occipital- und Mastoidgegend bis zur Mitte des gleichseitigen Kopfnickers einnimmt. Man nimmt nirgends eine Fluktuation wahr. Operation abgelehnt. Warme Umschläge.

2. IV. 1908. Deutliche Fluktuation an der Warzenfortsatzgegend; Wildesche Inzision. Nach Entleerung des reichlichen Eiters wird eine $5 \mathrm{~cm}$ tief in die Mastoidzellen führende Knochenfistel aufgefunden, durch welche beim täglichen Druck auf die Schwellung des Kopfnickers eine reichliche Menge Eiters regelmäßig ausquillt. - Es liegt die Vermutung nahe, dab es sich hier um eine zweite Knochenfistel an der medialen Fläche des Warzenfortsatzes handelt, durch welche ein Senkungsabszeb am Halse durch den Druck von unten nach oben retrograd entleert werden konnte. - Nach einigen Tagen sistiente der Ohrausfluß, die retroaurikuläre Eiterung heilte aber nach einigen Retentionserscheinungen erst nach $1 \frac{1}{2}$ Monat ohne weiteres definitiv aus.

8. Fräul. A. G., 16 Jahre alt, erkrankte am 9. XII. 1908 an Influenza mit Halsschmerzen und Temperaturerhöhung von $38^{\circ}-39^{\circ}$, welehe 4 Tage lang andauerte. Nach 4 tägigem Wohlbefinden Ohrenschmerzen rechts, die allmählich heftig wurden und erst einige Tage später nach spontanem Ohrenflub nachließen. Die Temperatur schwankte bis zum 5. I. 1909 zwischen $38^{\circ}-40^{\circ}$, der allgemeine Zustand verschlimmerte sich, und es traten Ohnmachtsanfälle und Frösteln ein, daneben rechtsseitige Kopfschmerzen und eine harte schmerzhafte Schwellung, welche vom hinteren Rande des Warzenfortsatzes beginnend sich nach dem Nacken zu ausbreitete. Beim Druck auf dieselbe empfindet Pat. einen Anprall im Ohr. Das Planum mast. gar nicht, dagegen die Warzenfortsatzspitze stark druckempfindlich, aber ohne Schwellung. Im Gehörgang viel Eiter; Trommelfellperforation nach hinten unten.

13. I. 1909. Im Österreich-Ungarischen Spital senkrechter, $4 \mathrm{~cm}$ langer Schnitt neben dem hinteren Rande des Warzenfortsatzes, durch dessen fistulös durchbrochene mediale Fläche eine passend gebogene Sonde in die Mastoldzellen ziemlich tief eindringt. Danach fließt reichlich blutig tingierter Eiter aus dem Gehörgange.

17. I. 1909. Pat. wurde ohne Beschwerden (OhrenfluB, Kopfschmerzen und Fieber) vom Spital entlassen.

30. I. 1909. Inzisionswunde seit mehreren Tagen zugeheilt; Gehör normal, Warzenfortsatzgegend nirgends empfindlich.

9. Herr J. S., 65 Jahre alt. Im Oktober 1909 erkrankte Pat. an Ohrenschmerzen links; eine anschließende Ohreiterung dauerte bis Februar 1910. Jetzt entwickelte sich eine schmerzhafte Schwellung hinter der Ohrmuschel, die von einem Kollegen nach Wilde gespalten wurde. Pat. suchte, da die Wunde nicht heilen wollte und beständig Eiter sickerte, das Griechisch-Nationalspital auf, wo er 20 Tage lang erfolglos behandelt wurde. Nach meiner Empfehlung wurde die Knochefistel, in deren Tiefe weiche Granulationen $\mathrm{zu}$ fühlen waren, mit Lapisperle geätzt, worauf die Eiterung sowohl aus der Fistel wie auch aus dem Gehörgang in einigen Tagen definitiv versiegte. Darauf Entlassung des Pat. aus dem Spitale. 
Im Mai 1910 Ohrenschmerzen rechts. Die darauf folgende profuse Ohreiterung wurde mit steriler Borsäurelösung ausgespült und in einigen Tagen ausgeheilt. Nachher rechtsseitige Kopfschmerzen und eine fluktuierende Schwellung auf der gleichseitigen Warzenfortsatzgegend, welche nach Wilde (14. Juli 1910) gespalten wurde. Nach Entleerung des Abszesses wurde eine Kortikalisfistel aufgefunden, welche tief in die Mastoidzellen führte. Die Nachbehandlung bis zur vollständigen Heilung dauerte eine Woche.

10. Herr B. J., 30 Jahre alt. Wegen einer rechtsseitigen Mittelohrentzündung wurde bei ihm Ende Januar 1904 die Trommelfellinzision und bei wiederkehrenden Retentionserscheinungen den 5. III. 1904 die Aufmeißlung vorgenommen, wobei auch die mittlere Schädelgrube und das Antrum eröffnet wurdea. Die Operationshöhle heilte in $1 \frac{1}{2}$ Monat zu, nacholem schon früher der Ohrausfluß sistiert hatte. Aber nach 2 jährigem Wohlbefinden sind $4 \mathrm{mal}$ Abszeßbildungen in der Narbe entstanden und nach Wilde eröffnet worden. Da die Wunde trotzdem beständig sickerte und nicht zuheilen wollte, so entschloß ich mich, den Pat. am 25. VI. 1906 nach Siebenmann mit Entfernung der hinteren Gehörgangswand und Erhaltung des Trommelfells zu operieren, wobei sich nur geringfügige Granulationsbildung im Antrum fand; nirgends ein Granulationskanal. Die ungestörte Nachbehandlung dauerte bis Ende Juli. Trotzdem im Februar 1908 abermalige Abszeßbildung in der Narbe. Der AbszeB wurde von neuem nach Wilde eröffnet und entleert und diesmal eine Lapisperle bis zum Antrum eingeführt ${ }^{1}$, was 2 mal in 3 tägigen Intervallen wiederholt wurde. Seit $1 \frac{1}{2}$ Jahr ist Pat. bis heute rezidivfrei geblieben.

11. Herr G. M., 45 Jahre alt. Vor ungefähr 3 Monaten hatte Pat. an linksseitigen Ohrenschmerzen gelitten, welche nach stattgehabtem Ohrenfluß nachgelassen hatten und nach Politzers Verfahren wiederkehrten. Allmählich bildete sich eine Schwellung in der Gegend rer Warzenfortsatzspitze, welche nach Ohrenfluß zurickging. Pat. klagt nun über Obrenfluß und über spontane Schmerzen um die Ohrmuschel, am Nacken und an der Temporalgegend links. Keine Schwellung an der Regio mast. Die Warzenfortsatzspitze leicht druckempfindlich. Uhr a. c. Gehörgang durch eine Granulation an der knöchernen und knorpeligen Grenze verengt; dortselbst fistulöser Knochen. Trommelfell verdickt, leicht gerötet, nicht vorgewölbt. Bei der AufneiBlung (24. VI. 1903) fanden sich eine Knochenfistél an der hinteren Gehörgangswand, einige eiternde Spitzenzellen und ein sehr enges, bis zur Dura der mittleren Schädelgrube führendes Granulationskanälchen. Einige Granulationen auf der Dura wurden abgeschabt und das Antrum eröffnet. Nach 5 wöchentlicher Nachbehandtung schloß sich die Wunde definitiv. Trotz Katheterismus besserte sich das, Gehör nicht.

10. X. 1909. Seit einigen Tagen schmerzhafte fluktuierende Schwellung der vor 6 Jahren operierten Gegend. Spaltung derselben nach Wilde, Entleerung eines großen Abszesses und Heilung ohne weiteres in einigen Tagen.

1) Ich hatte dieses Verfahren auch bei einem die Frau eines Kollegen betreffenden nach Killiam-Dahmer vor 3 Jahren operierten Fall von Sinuitis frontalis chronica exulcerans mit gutem Erfolg angewendet. 4 Monate nach der gut ausgeheilten Stimmhöhlenoperation entstand eine schmerzhafte fluktuierende Schwellung in der operierten Gegend, welche in der alten Narbe $1 \mathrm{~cm}$ breit gespalten wurde. Nach der Entleerung des reichlichen Eiters ätzte ich die Höhle hinter der Knochenspange 3 mal mit Lapisperle, wonach die Kiterung bis jetzt. sistierte und die Wunde schnell zuheilte. 
Ich halte es für unnötig und zeitraubend, über weitere 60 ähnliche, meistenteils Kinder betreffende Fälle zu berichtexณ Fast alle diese Mastoiditiden sind im Verlaufe von Infektioskrankheiten aufgetreten und zeigten schwere Veränderungen des Knochens; sämtliche sind durch den Wildescher Schnitt ausgeheilt, und niemals bin ich genötigt gewesen die Aufmeißelung bei denselben vorzunehmen. Dieselben xigen genügend, daß die oben angeführte Polemik der Autoren gegen die Wildesche Inzision nicht hinreichend begründet ist.

Von den oben erwähnten 11 Fällen bei Erwachsenen ist Nr. 8 als sicherer und Nr. 7 als verdächtiger Fall von Bezoldscher Mastoiditis aufzufassen. Man findet auch noch 2 Fälle (Nr.10 und 11) von rezidivierender Mastoiditis. Ob die Rezidivfreiheit dieser letzten Fälle von Dauer sein wird, wird die Zukunft zeigen.

Der Wildesche Schnitt ist also bei allen akuten Mastoiditiden mit Periostitis mast. indiziert. Gelingt es einmal den Prozeß damit nicht zur Heilung zu bringen, so kann man später zur Aufmeißelung schreiten. Man muß dazu 1 -2Monate warten, vorausgesetzt, daß keine Symptome einer lebensgefährlichen Komplikation vorliegen. Die meisten meiner Fälle sind in einer Woche geheilt worden; nur einer, Nr. 7, mußte 11/2 Monat unter einigen Retentionserscheinungen nachbehandelt werden.

Es kann auch jeweils vorkommen, daß der Wildesche Schnitt bei der akuten Mastoiditis mit Periostitis mast. versagt; hier steckt in der Tiefe ein Sequester, oder ein gewundenes Granulationskanälchen führt zu einer intrakraniellen. Komplikation, oder der Prozeß ist tuberkulöser Natur. Aber selbst bei der Aufmeißelung ist der auserlesenste Operateur nicht immer imstande, den ganzen Krankheitsprozeß zu eliminieren; verborgene Granulationskanäle oder aberrierte eiternde Mastoidzellen, die bei der Operation übersehen werden, machen nicht selten hier auch eine zweite Aufmeißelung erforderlich.

In den chronischen Fällen bin ich nicht ebenso glücklich wie Stetter gewesen; in den wenigen Fällen, bei denen ich die Wildesche Inzision anwendete, habe ich bloß nach Linderung und Aufhören des heftigsten Schmerzes getrachtet. Die chronische Mittelohreiterung ist hier nur durch die radikale Freilegung der Mittelohrräume gewichen.

Bei den Mastoiditiden ohne Periost. mast. ist der Wildesche Schnitt ebenso wie die üblichen Blutegel zu widerraten. Durch die Empfindlichkeit derartiger Hautwunden wird die Kontrollierung der Krankheit erschwert oder unmöglich ge- 
macht. Bei bedeutender Druckempfindlichkeit kann man hier einige Tage lang warten, bis die Kortikalis günstigenfalls durchbricht und sich ein Subperiostalabszeß bildet; dann ist die Wildesche Inzision indiziert. Ist aber die Druckempfindlichkeit an den hinteren Grenzen des Processus mast. gelegen es handelt sich dann meistenteils um eine Komplikation in der hinteren Schädelgrube - oder stellen sich wiederholte Retentionserscheinungen ein, so darf man selbst bei fehlender Druckempfindlichkeit nicht lange mit der Aufmeißelung zögern. Gewöhnlich handelt es sich im letzteren Falle um eine dicke kompakte Kortikalis, welche den Krankheitsprozeß in der Tiefe hält und ein Fortkriechen desselben ins Endokranium erleichtern kann.

Wenn ich mit der Wildeschen Inzision 100\% Heilungen bei der akuten Mastoiditis mit Periostitis mast. erreicht habe, so ist sie bei solchen Fällen nicht zu widerraten. Wird sie fleißig bei geeigneten Fällen benützt, so wird sie sich sehr ersprießlich erweisen und größere Operationen und konservative lokale Entstellungen den Patienten ersparen. Des bedachtsamen Mediziners heiligstes Ziel sei der größte Gewinn durch das mildeste Arzneimittel. 